\title{
Monitoreo hemodinámico invasivo por catéter de arteria pulmonar Swan-Ganz: conceptos y utilidad
}

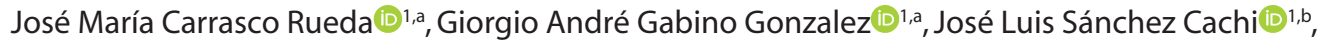

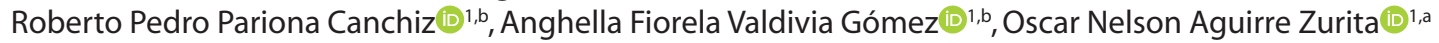

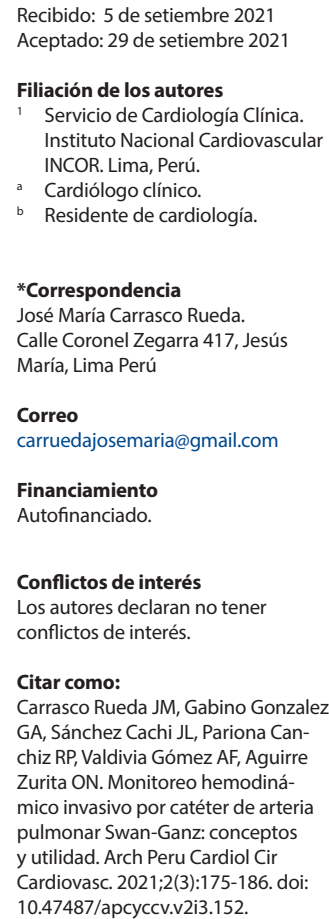

\section{RESUMEN}

Desde sus inicios en el siglo pasado, el cateterismo de arteria pulmonar (CAP) ha ido evolucionando hasta ser una técnica de evaluación hemodinámica invasiva que puede realizarse en la cama del paciente a través de un catéter Swan-Ganz; este procedimiento ha mantenido un curso intermitente en cuanto a su uso; no obstante, actualmente ha demostrado relevancia en escenarios específicos. El CAP permite el acceso a la circulación venosa central, el corazón derecho y la arteria pulmonar; realiza el cálculo de variables hemodinámicas de manera directa o indirecta mediante fórmulas y métodos establecidos. Esto hace posible realizar una evaluación y clasificación hemodinámica adecuada, realizar pruebas específicas (p. ej. prueba de vasorreactividad), que ayudan a definir el diagnóstico, la conducta terapéutica, monitorizar la respuesta al tratamiento, la evaluación previa a terapias avanzadas (p. ej. el trasplante cardiaco o dispositivos de asistencia circulatoria mecánica), y el pronóstico en los pacientes. En este artículo profundizamos sobre los conceptos y la utilidad del cateter de arteria pulmonar.

Palabras clave: Hipertensión pulmonar; Monitoreo hemodinámico; Choque cardiogénico; Insuficiencia cardiaca (fuente: DeCS BIREME).

\section{ABSTRACT}

\section{Invasive hemodynamic monitoring by Swan-Ganz pulmonary artery catheter: concepts and utility}

Since its beginnings in the last century, pulmonary artery catheterization (PAC) has evolved into an invasive hemodynamic evaluation technique that can be performed at the patient's bedside through a Swan-Ganz catheter; this procedure has maintained an intermittent course in terms of its use; however, it has currently demonstrated relevance in specific scenarios. The PAC allows access to the central venous circulation, the right heart and the pulmonary artery; it performs the calculation of hemodynamic variables directly or indirectly by means of established formulas and methods. This makes possible to perform an adequate hemodynamic evaluation and classification, perform specific tests (e.g. vasoreactivity test), which help to define the diagnosis, therapeutic, monitor the response to treatment, evaluation prior to advanced therapies (e.g. cardiac transplantation or mechanical circulatory assistance devices), and prognosis in our patients. In this article we discuss the concepts and usefulness of pulmonary artery catheterization.

Keywords: Hypertension, pulmonary; Hemodynamic monitoring; Cardiogenic shock; Heart failure (source: MeSH NLM). 


\section{Introducción}

El primer cateterismo cardíaco fue realizado en 1920 por el doctor Werner Forssmann a través de la introducción de una sonda urológica en la vena antecubital hasta la aurícula derecha (1). Posteriormente se realizaron rutinariamente cateterismos con dispositivos semirrígidos, y en 1953 Lategola y Rahn innovaron por experimentación animal, un sistema de catéter con balón para facilitar el uso de cateterismo derecho y pulmonar sin fluoroscopia asistida ${ }^{(2)}$.En 1970 Harold James Swan y William Ganz, como resultado de la serendipia y basados en los antecedentes descritos, crearon el sistema de catéter flexible con balón distal inflable en humanos teniendo como motivación el desarrollo de una técnica para el cuidado y estudio de pacientes cardiópatas agudos en quienes la fluoroscopia no estaba disponible o se encontraban en condiciones de inamovilidad por inestabilidad hemodinámica u otras causas ${ }^{(3,4)}$.

El cateterismo de arterial pulmonar (CAP) por catéter Swan-Ganz (CSG) fue considerado un método revolucionario en su momento; sin embargo, en años subsiguientes, con el avance de técnicas diagnósticas y terapéuticas, tuvo un declive en su uso. En la última década su utilidad volvió a tener protagonismo como monitoreo invasivo hemodinámico en pacientes con falla cardiaca, asociado a una disminución de la mortalidad y un aumento de la estancia hospitalaria ${ }^{(5-7)}$. Asimismo, su uso ha tomado relevancia en pacientes con choque cardiogénico (CC) hospitalizados en unidades de cuidado intensivo ${ }^{(8)}$.

El presente artículo tiene como objetivo realizar una revisión de la literatura actual sobre el concepto, uso, indicaciones y utilidad del CAP mediante CSG, así como la interpretación de los parámetros hemodinámicos cardiopulmonares.

\section{Concepto}

El catéter de arteria pulmonar es un catéter dirigido por flujo con punta de balón que permite un acceso rápido a la circulación venosa central, al corazón derecho y a la arteria pulmonar (AP) ${ }^{(1,9)}$. Su longitud es de aproximadamente $110 \mathrm{~cm}$, con un diámetro externo estándar de 7 o 7,5 French. El balón en la punta, cuando se infla, guía el catéter desde las venas intratorácicas mayores a través de la aurícula derecha (AD) y las cámaras ventriculares hacia la $\mathrm{AP}^{(9,10)}$. La mayoría tiene cuatro lúmenes separados, cada uno de los cuales tiene funciones individuales $(9,10)$ :

- El lumen proximal (azul) se encuentra en la $A D$ y mide la presión intraauricular. También se puede utilizar para administrar medicamentos.
- El lumen distal (amarillo) se encuentra en el extremo distal y reside en la AP. Se utiliza para controlar las presiones y para obtener una muestra venosa mixta.

- El puerto rojo es para inflar y desinflar el balón. Cada catéter está acompañado de una jeringa de 1,5 mL que se utiliza para inflar el balón.

- El sensor de temperatura (termistor) se usa para medir la temperatura central en la AP.

El objetivo del CAP es la monitorización hemodinámica y sus parámetros fisiológicos derivados de la evaluación de la función ventricular izquierda y derecha ${ }^{(1,89)}$. La técnica de termodilución que calcula el gasto cardiaco (GC) mide la variabilidad de temperatura sanguínea de la AP y la de la solución salina inyectada por $\mathrm{AD}$ que produce un cambio de resistencia y voltaje generando una curva de tiempo-temperatura a partir de la cual se estima el GC mediante la ecuación de Stewart-Hamilton ${ }^{(10)}$. Si el área bajo la curva es pequeña, la temperatura se equilibra rápidamente con la temperatura corporal ambiental lo que indica un GC alto, y si el área bajo la curva es grande implica un GC bajo ${ }^{(11,12)}$.

\section{Colocación}

Previo al procedimiento, se debe tener clara la indicación de colocación del CSG, descartar cualquier posible contraindicación y valorar el riesgo de complicaciones (Tabla 1) ${ }^{(9,11)}$. El introductor delCSG debe ser colocado percutáneamente vía vena cava inferior a través de las venas femorales o vía vena cava superior a través de la vena subclavia o yugular interna (acceso interfascicular), estos últimos accesos son de elección para el manejo a la cabecera del paciente. El abordaje interfascicular derecho por vena yugular interna es el preferido debido al fácil y rápido acceso a la AD. La punción se debe realizar en posición supina o Trendelenburg con guía ecográfica, confirmando un adecuado retorno venoso, y dilatando la zona de acceso a través de un dilatador para facilitar el ingreso del introductor venoso ${ }^{(13-15)}$.

El CSG debe ser introducido hasta aproximadamente 15 cm y obtener ondas de presión de AD, luego de ello, el balón distal será inflado y se continuará el ingreso rápido del catéter a través del ventrículo derecho (VD), para evitar ectopias ventriculares, hasta obtener ondas de presión de AP. Luego se progresará lentamente hasta obtener una curva de presión capilar pulmonar (PCP), esto sucede a los 50 o $55 \mathrm{~cm}$ de ingresado el cateter (Figura 1) ${ }^{(15)}$.

La puesta a cero del sistema de presiones es importante para obtener un valor adecuado de la PCP y se realiza posicionando el transductor a nivel de AD (línea medio-axilar, 4to espacio intercostal). Se debe fijar el CSG con el capuchón de protección para mitigar el riesgo de infecciones y se confirmará la adecuada posición localizando radiológicamente el catéter distal en la zona pulmonar III de West ${ }^{(9,14,15)}$. 
Tabla 1. Indicaciones, contraindicaciones y complicaciones del Catéter Swan-Ganz

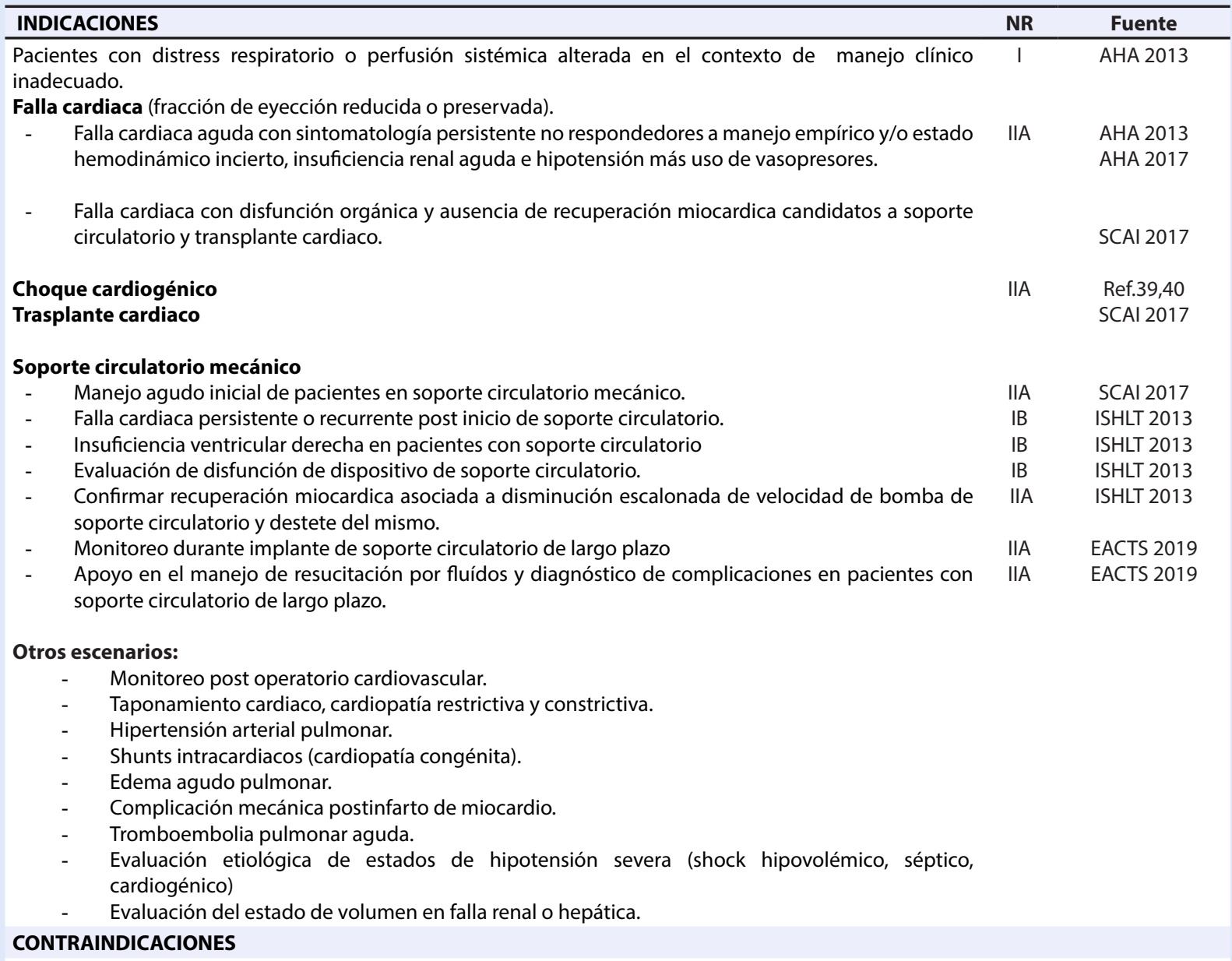

\section{Absolutas:}

Prótesis mecánica valvular tricuspídea o pulmonar, masa intracavitaria en ventrículo derecho, endocarditis derecha.

\section{Relativas:}

Presencia de marcapaso endocárdico, Prótesis biológica valvular tricuspídea o pulmonar, Sindrome de distress respiratorio secundario a sepsis pulmonar,Bloqueo completo de rama izquierda del haz de His, arritmias significativas, infección de zona de punción.

\section{COMPLICACIONES}

Hemo/neumotórax, arritmias, trastorno de conducción, hematoma, hipotensión, evento vasovagal, sangrado de vías respiratorias bajas, infección de sitio de inserción de catéter.

AHA: American Heart Association. SCAI: Society for Cardiovascular Angiography \& Interventions. EACTS: Expert Consensus on long-term mechanical circulatory support. ISHLT: International Society for Heart \& Lung Transplantation. NR: nivel de recomendación.

Adaptado de Hsu S, Fang JC, Borlaug BA. Hemodynamics for the Heart Failure Clinician: A State-of-the-Art Review [published online ahead of print, 2021 Aug 8]. J Card Fail. 2021;S1071-9164(21)00306-7. doi:10.1016/j.cardfail.2021.07.012 y Madias C and Kimmelstiel C. Right Heart Catheterization. Cardiology Procedures. USA: Springer; 2017: $123-133$.

El GC por termodilución, se realiza infundiendo $10 \mathrm{~mL}$ de suero salino por el lumen proximal con una jeringa en menos de 4 segundos. La modificación de la constante de cálculo de $\mathrm{GC}$ depende de la temperatura de infusión, siendo 0,532 para $0{ }^{\circ} \mathrm{C}$ y 0,586 para $24^{\circ} \mathrm{C}$ (esto puede variar según la marca y tipo de monitor). Con el GC obtenido se realizarán los cálculos hemodinámicos, se debe tener en cuenta que la presencia de insuficiencia tricuspídea, estados de bajo gasto o shunts intracardiacos pueden alterar la exactitud de los resultados ${ }^{(9,11,15)}$.

\section{Monitoreo hemodinámico}

\section{Aurícula derecha}

El trazado de presión de la $A D$ presenta diferentes curvas de presión (secuencia A-X-V-Y). La onda A representa la contracción auricular, el descenso $\mathrm{X}$ la caída de presión durante la sístole ventricular temprana y la relajación auricular; la onda V el llenado auricular durante la sístole ventricular y 
el descenso $Y$ la diástole temprana con el vaciamiento rápido de la AD. Así, el descenso X y la pendiente ascendente de la onda $V$ son eventos sistólicos, mientras que el pico de la onda $V$, el descenso $Y$ y la onda A son eventos diastólicos. La presión venosa central (PVC) refleja la presión intrauricular derecha, se prefiere su medición a nivel del punto $Z$ representado por la correlación del inicio del segmento QRS en el ECG y la intersección entre la onda A y C. La PVC manifiesta el estado volémico del paciente y su relación directa con el VD, se puede interpretar como las presiones de llenado del lado derecho del corazón (Tabla 2) ${ }^{(16)}$.

\section{Ventrículo derecho}

Los trazos del VD exhiben un rápido aumento de la presión durante la contracción ventricular y un rápido descenso de la presión durante la relajación, con una fase diastólica caracterizada por una presión inicialmente baja que aumenta gradualmente. La presión de la $A D$ debe ser bastante cercana a la presión telediastólica del VD, a menos que exista estenosis tricuspídea. Con la contracción auricular, puede aparecer una onda $\mathrm{A}$ al final de la diástole ventricular, siendo un hallazgo anormal y suele indicar una distensibilidad disminuida, como en pacientes con hipertensión pulmonar (HTP), hipertrofia del VD o sobrecarga de volumen.

La distensibilidad de arteria pulmonar (DAP), determinado por los componentes resistivos y pulsátiles de la carga del VD, representa la relación entre el volumen eyectivo y la presión de pulso pulmonar (PPP). Ha demostrado ser un fuerte indicador pronóstico de mortalidad y disfunción de VD en HTP tipo Il y falla cardiaca con fracción de eyección reducida (FCFEVIr);

Tabla 2. Parámetros de medición directa en el monitoreo hemodinámico por catéter Swan Ganz

\begin{tabular}{|c|c|c|}
\hline Nombre & Rango normal & Descripción \\
\hline $\begin{array}{l}\text { Presión venosa central } \\
\text { (PVC) }\end{array}$ & $2-6 \mathrm{mmHg}$ & $\begin{array}{l}\text { Representa a la presión de aurícula derecha, e interpreta las presiones de llena- } \\
\text { do del lado derecho del corazón. } \\
\text { PVC > } 15 \mathrm{mmHg} \text { indica sobrecarga de presiones del lado derecho. }\end{array}$ \\
\hline Presión de VD (PVD) & $\begin{array}{l}\text { VDs: } 15-30 \mathrm{mmHg} \\
\text { VDd } 2-8 \mathrm{mmHg}\end{array}$ & $\begin{array}{l}\text { Posee una onda sinusoidal de rápido ascenso y descenso. La presencia de una } \\
\text { onda } \mathrm{A} \text { al final de la diástole suele indicar una distensibilidad disminuida. }\end{array}$ \\
\hline Presión de AP (PAP) & $\begin{array}{l}\text { PAs } 15-30 \mathrm{mmHg} \\
\text { PAd } 8-15 \mathrm{mmHg} \\
\text { PAm } 14-16 \mathrm{mmHg}\end{array}$ & $\begin{array}{l}\text { Presenta un rápido ascenso y lento descenso con una muesca dicrota, que repre- } \\
\text { senta el cierre de la válvula pulmonar. } \\
\text { PAPs alta asociada a una frecuencia cardiaca elevada es indicativo de disfunción } \\
\text { ventricular derecha y alta incidencia de eventos cardiacos. }\end{array}$ \\
\hline $\begin{array}{l}\text { Presión cuña capilar } \\
\text { (PCP) }\end{array}$ & $6-12 \mathrm{mmHg}$ & $\begin{array}{l}\text { La forma de la onda es similar en apariencia a la onda de presión de la aurícula } \\
\text { derecha con algunas diferencias (mayor variabilidad con el ciclo ventilatorio y la } \\
\text { magnitud de la onda «V» supera a la onda «a» en el trazado). } \\
\text { Si PCP }>15 \mathrm{mmHg} \text { indica edema hidrostático, se debe corregir la PCP si el PEEP } \\
\text { es alto y }>10 \mathrm{mmHg} \text {. }\end{array}$ \\
\hline $\begin{array}{l}\text { Gasto cardíaco (GC) } \\
\text { Índice cardíaco (IC) }\end{array}$ & $\begin{array}{c}4-8 \mathrm{~L} / \mathrm{min} \\
2.5-4 \mathrm{~L} / \mathrm{min} / \mathrm{m} 2\end{array}$ & $\begin{array}{l}\text { Calculable por termodilución mediante la ecuación de Stewart-Hamilton y por } \\
\text { principio de Fick, preferible el cálculo por termodilución salvo presencia de } \\
\text { shunt intracardiaco de izquierda a derecha donde el uso de Fick es recomenda- } \\
\text { ble. } \\
\text { GC }<4 \text { L/min indica escenario de bajo gasto cardíaco. } \\
\text { IC }<2,2 \text { criterio diagnóstico de choque cardiogénico. }\end{array}$ \\
\hline Aurícula izquierda (Al) & $5-10 \mathrm{mmhg}$ & $\begin{array}{l}\text { Inversamente relacionada a la distensibilidad ventricular izquierda. La PCP se } \\
\text { asocia directamente a las presiones de Al y estas a su vez a la presión telediastó- } \\
\text { lica del VI. }\end{array}$ \\
\hline $\begin{array}{l}\text { Saturación venosa } \\
\text { mixta }(\mathrm{SvO} 2)\end{array}$ & $60-80 \%$ & $\begin{array}{l}\text { Análisis por oximetría de una muestra de sangre extraída de la arteria pulmonar } \\
\text { (lumen distal). } \\
\text { Saturación venosa central < } 60 \% \text { en infarto de miocardio indicativo de estado } \\
\text { de bajo gasto y choque cardiogénico. } \\
\text { Saturación venosa mixta < } 60 \% \text { es un indicador de hipoperfusión, acidosis } \\
\text { láctica y mal pronóstico. }\end{array}$ \\
\hline
\end{tabular}

HTP: hipertensión arterial pulmonar. Pas: presión arterial sistólica PAd: presión arterial diastólica. PAm: presión arterial media. PEEP: presión positiva al final de la espiración. VD: ventrículo derecho. VDd: presión diastólica de ventrículo derecho. VDs: presión sistólica de ventrículo derecho. VI: ventrículo izquierdo. 


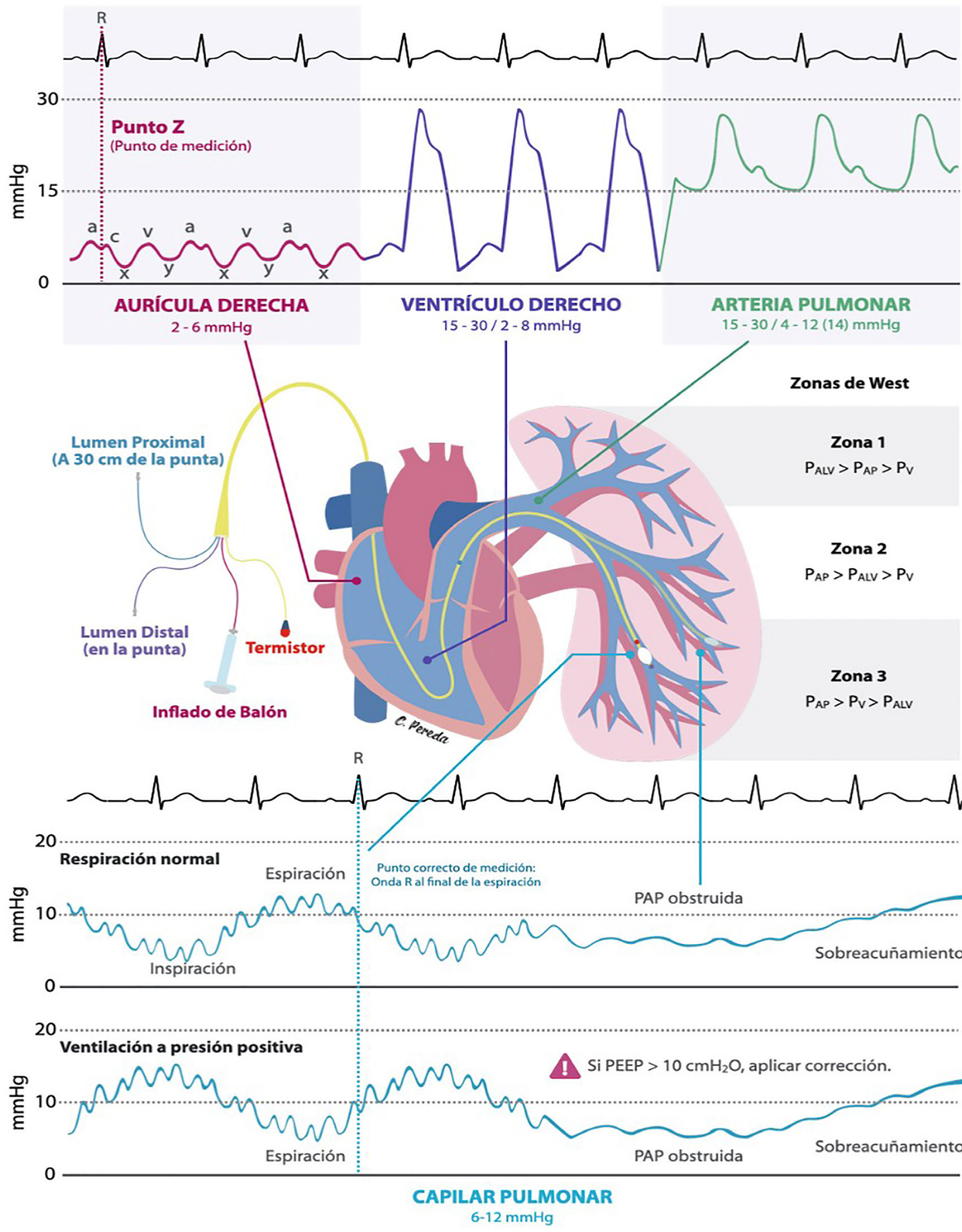

Figura 1. Monitoreo hemodinámico por catéter arterial pulmonar Swan-Ganz.

PAP: Presión de arteria pulmonar. PEEP: Presión positiva al final de la espiración.

un valor menor a 2,15 se asocia con una menor supervivencia, incluso en aquellos pacientes con RVP normal ${ }^{(17,18)}$.

Por otro lado, la elastancia efectiva de arteria pulmonar (EAP), medida que relaciona la presión sistólica de AP (PAPs) con el volumen eyectivo de Vl, representa al igual que la DAP la poscarga y función del VD y, por ello, es un factor predictivo más específico de mortalidad y disfunción de VD (independientemente de la RVP y la GDP) en pacientes con HTP tipo Il y FC incluso con FCFEVIp. Valores mayores a 1 apoyan la necesidad de una terapia dirigida a mejorar la carga total del VD en lugar del componente precapilar, y se recomienda su uso en pacientes con FC descompensada e HTP II, así como falla de VD ${ }^{(17,18)}$. 
Tabla 3. Interpretación de monitoreo hemodinámico según escenarios

\begin{tabular}{|c|c|c|c|c|c|c|c|}
\hline Escenario & PA & FC & AD & PCP & GC & RVS & Acciones \\
\hline & - & $-/ \uparrow$ & $-/ \downarrow$ & $-/ \downarrow$ & $-/ \downarrow$ & - & Detener descongestión aguda. \\
\hline $\begin{array}{l}\text { Estatus no claro de } \\
\text { volemia, perfusión y } \\
\text { resistencia vascular. }\end{array}$ & $-/ \uparrow$ & $-/ \uparrow$ & $\uparrow \uparrow$ & $\uparrow \uparrow$ & $\downarrow$ & $\uparrow \uparrow$ & $\begin{array}{l}\text { Diurético + vasodilatador. Mantener PVC }< \\
8 \mathrm{mmHg}, \mathrm{PCP}<15 \mathrm{mmHg} \text {, RVS } 1000 \text { a } 1200 \\
\text { dynas } / \mathrm{s} / \mathrm{cm}^{-5} \text { y PAM }>65 \mathrm{mmHg} .\end{array}$ \\
\hline $\begin{array}{l}\text { Deterioro de la } \\
\text { función renal durante } \\
\text { descongestión. }\end{array}$ & $\downarrow$ & $\uparrow$ & $\uparrow \uparrow$ & $\uparrow \uparrow$ & $\uparrow$ & - & $\begin{array}{l}\text { Inotrópicos }(P A M>65 \mathrm{mmHg} \text { e } \\
\left.\text { IC }>2 \mathrm{~L} / \mathrm{min} / \mathrm{m}^{2}\right)\end{array}$ \\
\hline \multirow[t]{2}{*}{$\begin{array}{l}\text { Disnea y estado no claro } \\
\text { de volemia. }\end{array}$} & - & - & 个个 & $-/ \downarrow$ & $-/ \downarrow$ & $-\backslash \uparrow$ & $\begin{array}{l}\text { Sospecha diagnóstica y manejo de infarto } \\
\text { de VD, HTP, pericarditis, TEP. }\end{array}$ \\
\hline & - & - & $\uparrow$ & 个个 & - & - & Diuréticos \\
\hline \multirow[t]{3}{*}{$\begin{array}{l}\text { Hipotensión de etiología } \\
\text { no definida. }\end{array}$} & $\downarrow \downarrow$ & $\uparrow$ & $-/ \downarrow$ & $-/ \downarrow$ & $\downarrow$ & $\uparrow \uparrow$ & Fluido IV (Probable choque hipovolémico) \\
\hline & $\downarrow \downarrow$ & $\uparrow$ & - & - & $-\backslash \uparrow$ & $\downarrow \downarrow$ & $\begin{array}{l}\text { Fluido IV, vasopresor, manejo etiológico. } \\
\text { (Probable choque distributivo) }\end{array}$ \\
\hline & $\downarrow \downarrow$ & $\uparrow$ & $\uparrow \uparrow$ & $\uparrow \uparrow$ & $\downarrow \downarrow$ & $\downarrow \backslash \uparrow$ & Inotrópico y vasopresor. Posibilidad de SMC. \\
\hline
\end{tabular}

AD: aurícula derecha. FC: frecuencia cardiaca. GC: gasto cardiaco. HTP: Hipertensión arterial pulmonar. IC: índice cardiaco. IV: intravenoso. PA: presión arterial. PAM: presión arterial media. PVC: Presión venosa central. PCP: presión capilar pulmonar. RVS: resistencia vascular sistémica. TEP: tromboembolia pulmonar. VD: ventrículo derecho.

Adaptado de: Hsu S, Fang JC, Borlaug BA. Hemodynamics for the Heart Failure Clinician: A State-of-the-Art Review [published online ahead of print, 2021 Aug 8]. J Card Fail. 2021;S1071-9164(21)00306-7. doi:10.1016/j.cardfail.2021.07.012

El índice de pulsatibilidad arterial pulmonar (PAPi) determinado por la relación entre la PPP y la $A D$, es un parámetro que predice la disfunción severa de VD en el contexto de un infarto de miocardio inferior y/o soporte circulatorio mecánico (SCM) de VI, desarrollado con el fin de identificar pacientes con requerimiento de asistencia mecánica derecha; asimismo, tiende a ser más predictivo en pacientes con soporte inotrópico y es útil como indicador pronóstico de supervivencia de HTP cuando los valores son $<0,95^{(19,20)}$.

\section{Arteria pulmonar}

El trazado de la presión de la AP muestra un rápido aumento de la presión, un pico sistólico, una disminución de la presión después de la eyección máxima y una muesca dicrótica bien definida por el cierre de la válvula pulmonar durante la disminución de la presión. No debe existir una diferencia de presión sistólica entre el ventrículo derecho y la AP, a menos que exista estenosis de la arteria o válvula pulmonar.

La forma de onda de la AP, al igual que otras formas de onda de presión del corazón derecho, está sujeta a cambios respiratorios, por ello los pacientes en ventilación mecánica, con enfermedad pulmonar grave, obesidad mórbida o dificultad respiratoria, pueden generar cambios sustanciales en la presión intratorácica con diferencias marcadas en las presiones de la AP durante las fases respiratorias. La mayoría de los expertos consideran que el final de la espiración es el punto adecuado para evaluar las presiones de la arteria pulmonar (y de otras cámaras cardíacas) porque es en esta fase donde la presión intratorácica se encuentra más cerca de cero ${ }^{(21)}$.

La PAPs se produce al mismo tiempo que la onda T en el ECG y ha demostrado ser un parámetro relacionado a eventos cardíacos mayores. Una PAPs asociada a una frecuencia cardiaca alta determina un mayor consumo de oxígeno por el VD en HTP tipo l y, por ende, mayor riesgo de disfunción de VD ${ }^{(22)}$.

La medición de la presión arterial pulmonar media (PAPm) es relevante en el diagnóstico y manejo de HTP. Según el último consenso del Sexto Simposio Mundial de Hipertensión Pulmonar (WSPH), un valor $>20 \mathrm{mmHg}$ representa un punto de corte para el diagnóstico de HTP ya que el manejo médico adecuado a partir de este valor muestra beneficios en la sobrevida ${ }^{(23)}$.

\section{Presión capilar pulmonar}

La PCP es medida directamente en ausencia del flujo anterógrado de la AP, de modo que se transmita desde la aurícula izquierda, 
a través de las venas pulmonares y el lecho capilar pulmonar. Normalmente se encuentra a unos milímetros de mercurio por debajo de la presión de la aurícula izquierda ( 0 a $5 \mathrm{mmHg})^{(24,25)}$. Las características de una buena onda incluyen la presencia de ondas A y V, la confirmación fluoroscópica de la ubicación de la punta del catéter en AP distal con el globo inflado, la observación de una curva PAPm cuando se desinfla el balón o se retira el catéter de su posición, y una saturación de oxígeno con el globo inflado > 90\%, siendo esta última la más específica (Tabla 2). Si la punta del catéter se encuentra mal posicionada en una rama periférica de AP con el globo sobre distendido se produce un fenómeno de «sobreacuñamiento» observándose una curva de PCP falsa con una línea oscilante sin ondas A y V, lo cual puede provocar la ruptura abrupta de la $\mathrm{AP}^{(25)}$.

En pacientes con ventilación mecánica con presión positiva al final de la espiración (PEEP) $>10 \mathrm{cmH} 2 \mathrm{O}$ se produce un aumento significativo de la presión alveolar, lo cual reduce la proporción de la zona pulmonar 3 de WEST, siendo afectadas directamente las presiones del lado derecho provocando una sobreestimación de la PCP ${ }^{(25)}$. La corrección directa de la PCP por PEEP elevado se realiza sustrayendo de la PCP la presión esofágica medida con un balón intraesofágico; no obstante, existen otros métodos prácticos como la sustracción de 2 a $3 \mathrm{mmHg}$ de PCP por cada $5 \mathrm{cmH} 2 \mathrm{O}$ de aumento del PEEP o argumentando que la PCP corregida es igual al PCP medido menos la mitad del cociente del PEEP dividido por $1,36^{(16,25,26)}$.

Si la PCP está elevada, el aumento de la relación entre AD y PCP sirve como indicador de disfunción de VD e incremento de complicaciones en IC avanzada, su valor se asocia con mayor resistencia pulmonar y mortalidad intrahospitalaria ${ }^{(26)}$.

Para evaluar si el aumento de la presión de AP es secundaria a una elevación aislada de la presión de enclavamiento pulmonar, clásicamente se utilizaba la gradiente de presión arterial transpulmonar cuyo valor varía según el flujo de PAPm y las presiones de llenado de Vl; actualmente, su relevancia ha disminuido notablemente. Sin embargo, la gradiente de presión arterial pulmonar diastólica es un marcador más exacto que permite clasificar adecuadamente a la HTP según su componente pre y poscapilar; además, un valor elevado es considerado como predictor de mortalidad, mal pronóstico y hospitalización por falla cardiaca ${ }^{(24,27)}$.

Por último, la resistencia vascular pulmonar (RVP) es un marcador que desde el sexto WSPH ha ganado protagonismo; determinando la presencia de enfermedad vascular pulmonar al ser un mejor indicador de HTP precapilar, en contraste a la GDP (3). Es utilizado, además, como parámetro terapéutico en pacientes con cardiopatías congénitas y en pacientes en espera de un trasplante cardiaco (Figura 1) $24,27,28)$.

\section{Ventrículo izquierdo (VI)}

La presión del VI tiene una aceleración rápida durante la sístole inicial, seguida de un descenso rápido. La presión diastólica inicial es baja, mientras que la final aumenta lentamente hasta que la aurícula izquierda se contrae representando la verdadera precarga del ventrículo izquierdo ${ }^{(29)}$. Con la ayuda del CAP se puede calcular el GC y, por ende, el índice cardiaco (IC) mediante termodilución y por el principio de Fick ${ }^{(10)}$ (Figura 2). La presencia de un IC disminuido es el indicador hemodinámico principal en el diagnóstico de CC. Puede ayudar a reconocer un mal funcionamiento de los dispositivos de SCM y evaluar la recuperación del miocardio con miras al destete. Junto con la resistencia vascular sistémica (RVS) el IC permite diferenciar los distintos tipos de fenotipos de CC ${ }^{(6)}$. Con los valores del GC se puede calcular el poder cardíaco (PC) el cual es el marcador hemodinámico independiente más fuerte de mortalidad intrahospitalaria que el Shock trial registró dentro de la estratificación de riesgo en pacientes con infarto de miocardio, un valor $<0,6$ Watts es indicador de disfunción severa del VI ${ }^{(10,29)}$.

Por otro lado, la presión de llenado transmural de VI representa la precarga del VI, así como la diferencia entre la presión capilar pulmonar (PCP) y la presión pericárdica (PP), utilizando la PVC en lugar de la PP en el cálculo. La PCP varía con los cambios de presión transmural del VI y la PP, por lo que en un paciente con $\mathrm{FC}$ evidencia el efecto restrictivo pericárdico sobre la precarga del VI ${ }^{(11,30)}$.

Existe también una medida de valoración biventricular llamada índice de trabajo sistólico(iTS). Este parámetro evalúa el trabajo (energía) ventricular y ha demostrado que su caída en VI es un predictor de mal pronóstico y necesidad de mayor soporte hemodinámico; además, el iTS de VD es un predictor de falla de VD en SCM y mortalidad en pacientes postransplante pulmonar ${ }^{(31)}$ (Figura 2).

\section{Shunt intracardiaco (SI)}

Una saturación de oxígeno de AP mayor a 75\% puede indicar la presencia de un SI de izquierda a derecha, por lo que es recomendado realizar la medición de la vena cava superior e inferior, AD (media, alta y baja), VD y AP. El aumento de la saturación de oxígeno $\geq 7 \%$ puede ser indicativo de un shunt auricular de izquierda a derecha, mientras que $\geq 5 \%$ puede indicar un shunt a nivel del VD o de AP. Cuando se sospecha de un SI izquierda a derecha, el método directo de Fick es el medio preferido de medición del GC ${ }^{(32)}$. 

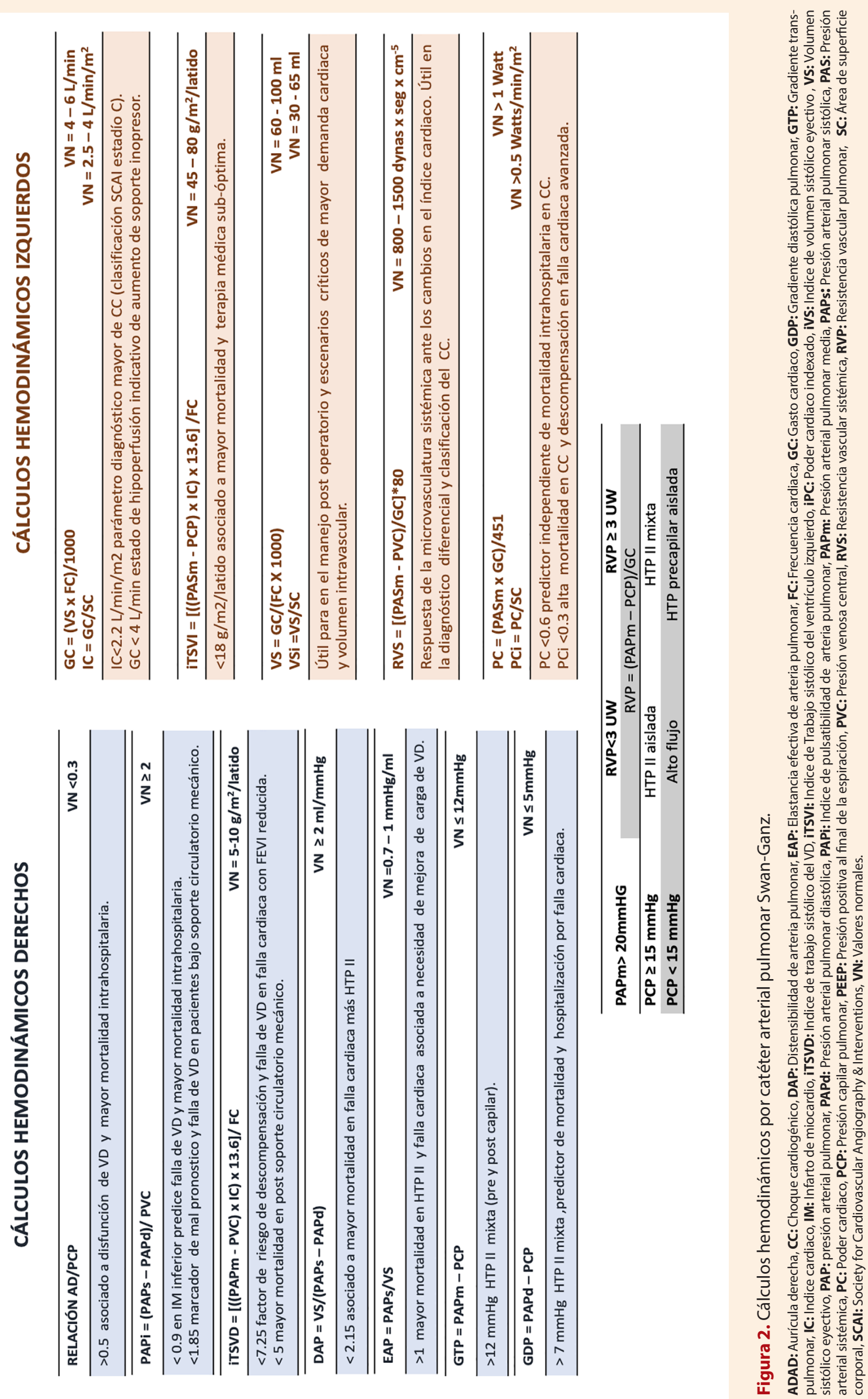


\section{Indicaciones y utilidad}

\section{Choque Cardiogénico}

Estudios actuales sugieren que el uso de CAP en CC se asocia con un beneficio en la morbimortalidad del paciente; actualmente, la Academia Americana de Cardiología (AHA) recomienda el uso temprano del CAP en el manejo de CC, sobre todo en casos de refractariedad a tratamiento $y / 0$ incertidumbre terapéutica ${ }^{(1,8,33,34)}$. Un extenso estudio retrospectivo observó que el uso de CAP en CC se asoció con menor mortalidad y menor incidencia de arresto cardiaco intrahospitalario comparado con aquellos en manejo convencional ${ }^{(35)}$. Otro estudio multicéntrico de similares características demostró que el manejo guiado por CAP ofrece una mayor sobrevida intrahospitalaria en los diversos escenarios de CC antes del inicio del SMC, debido a que permite reconocer el tipo de escenario, la necesidad de titulación de drogas y el momento de inicio de SMC ${ }^{(8,36)}$.

El reconocimiento del escenario y fenotipo hemodinámico del CC por CAP basado en la valoración del PC, PAPi y PDFVI, tiene un valor relevante en la guía de la estrategia de manejo hasta la recuperación del paciente o el uso de SMC; además, ayuda a prevenir las consecuencias hemometabólicas del estado de hipoperfusión y falla orgánica producidas por el CC (Tabla 3) $)^{(1,8,34)}$.

Dentro de estos fenotipos de presentación se describe al CC secundario a la falla del VD, que abarca el $30 \%$ de las presentaciones clínicas. En contextos como este, la Sociedad Europea de Cardiología (ESC) valora el uso del CAP en pacientes que no responden a terapias iniciales (Ilb C) o en caso de duda diagnóstica o terapéutica. La utilidad del CAP es fundamental para la correcta toma de decisiones en la preservación de un óptimo estado euvolémico y la decisión de manejo inotrópico y/o uso de SMC ${ }^{(36,37)}$.

Por otro lado, un subestudio del Shock trial reportó que en pacientes con CC, el SIRS presenta un predictor de muerte importante y se asocia con un aumento no significativo de muertes con valores de RVS bajo, por lo que el CAP puede ser fundamental al identificar este tipo de escenarios, permitiendo un manejo y seguimiento temprano adecuado ${ }^{(38)}$.

La utilidad de la medición de parámetros hemodinámicos por CAP en CC se ve manifiesta en el beneficio que poseen estos valores como predictores de mortalidad, tal como lo reporta el estudio CardShock, un registro prospectivo y multicéntrico que evidenció que el IC, IPC y el VS indexado son fuertes predictores de mortalidad a 30 días; asimismo, el manejo de CC guiado por CAP se asocia con un tratamiento más agresivo sin compromiso de la sobrevida a 30 días ${ }^{(25)}$. Por último, un estudio retrospectivo contemporáneo reportó una menor incidencia de mortalidad, stroke y readmisión hospitalaria relacionada con un mayor uso de SMC y trasplante cardiaco. Por lo expuesto, se concluye que el CAP en CC se asocia con un mayor beneficio clínico y a un mayor uso de terapias avanzadas ${ }^{(39,40)}$.

\section{Falla cardiaca}

El monitoreo hemodinámico por CAP de manera rutinaria no es recomendable en pacientes con FCFEVIr ya que, según el estudio ESCAPE, no ha demostrado beneficio sobre la mortalidad durante su uso rutinario inicial en pacientes con FC aguda descompensada ${ }^{(25)}$. En 2019, un estudio retrospectivo de amplia extensión evidenció una asociación entre el uso de monitoreo hemodinámico en pacientes con FC sin CC y mortalidad elevada; sin embargo, el mismo estudio describe una disminución significativa del índice de mortalidad en el tiempo asociado al aumento del uso de terapias avanzadas y SMC, donde el uso de CSG demuestra tener un mayor beneficio ${ }^{(37)}$. Su utilidad toma importancia en pacientes con FC cuyo estado de perfusión o volemia es incierto, sobre todo en casos de FC avanzada o CC, pues la literatura describe un beneficio en su uso al mejorar la supervivencia, disminuir las complicaciones e incentivar el uso oportuno de soporte avanzado ${ }^{(1,36,41)}$.

Por otro lado, aproximadamente la mitad de pacientes con FC poseen fracción de eyección preservada (FCFEVIp), su diagnóstico está basado en el uso de parámetros no invasivos; sin embargo, según la nueva guía 2021 de FC de la ESC, la prueba confirmatoria diagnóstica consiste en la evaluación invasiva de la PCP al reposo( $>15 \mathrm{mmHg}$ ) y al ejercicio(> $25 \mathrm{mmHg}$ ), aunque su uso es limitado y no posee una indicación rutinaria, el CAP ofrece un beneficio relevante cuando los marcadores no invasivos aportan una baja sensibilidad diagnóstica; además, cumple una función importante en el planteamiento de diagnósticos diferenciales, así como información adicional sobre las posibles entidades causantes de FCFEp ${ }^{(42,43)}$. El monitoreo hemodinámico al esfuerzo es útil ya que evalúa directamente la variabilidad de presiones, y aporta un valor predictivo al calcular la pendiente PCP/GC. En pacientes hospitalizados un aumento de la PCP con la maniobra de elevación de piernas es un buen discriminador de FCFEVIr y disnea no cardiaca ${ }^{(1,42)}$.

En casos de complicaciones mecánicas agudas, frecuentemente secundarias a infarto de miocardio, el CAP es usualmente no requerido, a excepción del contexto de un paciente con inestabilidad hemodinámica, progresión de la falla cardiaca posinfarto o la sospecha diagnóstica de un SI por ruptura del septum interventricular al obtener la saturación de oxígeno entre las cámaras cardiacas (AD, AP, VD); asimismo, el CAP puede servir de herramienta diagnóstica confirmatoria de SI al calcular la relación del flujo sistémico y pulmonar ${ }^{(44)}$. 


\section{Hipertensión pulmonar}

El CAP es útil en HTP pues determina directamente las presiones de AP. Se demostró que pacientes que se encontraban en una aparente zona gris (PAPm 21-24 mmHg) presentaban un incremento de la mortalidad, por lo que el $6^{\text {to }} \mathrm{WSPH}$ ha planteado un cambio en los puntos de corte para el diagnóstico de HTP $(\text { PAPm }>20 \mathrm{mmHg})^{(5,45)}$.

Según la ESC el CAP es el gold standard para confirmar el diagnóstico de HTP pues permite definir el perfil hemodinámico, la severidad, clasificación y el planteamiento de diagnósticos diferenciales ${ }^{(43)}$. Así mismo, permite realizar evaluaciones adicionales como la prueba de vasorreactividad o el monitoreo hemodinámico al esfuerzo, parámetros con gran relevancia durante la valoración del pronóstico, la orientación terapéutica y la respuesta a la misma.

Por otro lado el CAP es útil enel diagnóstico de la HTP inducida por ejercicio y necesaria para distinguirla de la FCFEVIp. Estudios recientes han demostrado que estos pacientes poseen peor pronóstico clínico; no obstante, el diagnóstico temprano e inicio precoz de tratamiento puede mejorar la sobrevida ${ }^{(1)}$.

\section{Dispositivos de asistencia ventricular izquierda (DAVI) y trasplante cardiaco (TC)}

El rol del CAP es fundamental en ambos escenarios. En la evaluación pre-TC la valoración del estado hemodinámico de los pacientes influye en la presencia de complicaciones como FC aguda o muerte pos-TC; la presencia de componente precapilar (RVP > 3,5 UW) es una contraindicación relativa, y su evaluación a través de una prueba de reversibilidad estratifica el perfil de riesgo ${ }^{(1,16)}$.

Durante la consideración del DAVI es importante una evaluación integral del VD con predictores hemodinámicos mediante CAP, lo cual permite valorar el riesgo de disfunción de VD ( $42 \%$ de casos), pues tiene importantes repercusiones en morbilidad y mortalidad. Además, durante el manejo posimplante permite una optimización más rápida del dispositivo en comparación con el seguimiento clínico-ecocardiográfico; así mismo, brinda una mejor precisión diagnóstica cuando se presentan complicaciones asociadas al dispositivo ${ }^{(9,32,46)}$.

\section{Otras patologías}

El CAP puede ser útil en pacientes con cardiopatía congénita y/o valvular cuando las evaluaciones no invasivas son inconclusas y para el diagnóstico diferencial entre pericarditis constrictiva y miocardiopatía restrictiva ${ }^{(1,9)}$.

\section{Conclusiones}

El CSG actualmente ha demostrado ser útil en el manejo de diversos escenarios tanto para pacientes con choque cardiogénico y falla cardiaca avanzada como pacientes con HTP, FC e incluso FCFEVIp debido a su utilidad diagnóstica y apoyo terapéutico.

El monitoreo porCSGy la interpretación de los parámetros hemodinámicos son una herramienta relevante en el tratamiento del paciente, capaz de apoyar en la decisión de brindar asistencia avanzada, SCM y TC, por lo que se está retomando protagonismo en el área cardiovascular.

\section{Contribución de los autores}

Todos los autores participaron en la elaboración, redacción y corrección del manuscrito, así como en la elaboración de figuras y tablas.

\section{Agradecimiento}

Agradecimiento y créditos al Dr. Carlos Manuel Pereda Joh por su aporte en la elaboración y edición del gráfico principal de este artículo.

\section{Referencias bibliográficas}

1. Hsu S, Fang JC, Borlaug BA. Hemodynamics for the Heart Failure Clinician: A State-of-the-Art Review. J Card Fail. 2021;510719164(21)00306-7. doi:10.1016/j.cardfail.2021.07.012

2. Lategola M, Rahn H. A self-guiding catheter for cardiac and pulmonary arterial catheterization and occlusion. Proc Soc Exp Biol Med. 1953;84(3):667-668. doi:10.3181/00379727-84-20745

3. Swan HJ, Ganz W, Forrester J, Marcus H, Diamond G, Chonette D. Catheterization of the heart in man with use of a flow-directed balloon-tipped catheter. N Engl J Med. 1970;283(9):447-51. doi:10.1056/NEJM197008272830902.
4. Forrester JS. A Tale of Serendipity, Ingenuity, and Chance: 50th Anniversary of Creation of the Swan-Ganz Catheter. J Am Coll Cardiol. 2019;74(1):100-103. doi:10.1016/j.jacc.2019.04.050.

5. Binanay C, Califf RM, Hasselblad V, O'Connor CM, Shah MR, Sopko G, et al. Evaluation study of congestive heart failure and pulmonary artery catheterization effectiveness: the ESCAPE trial.JAMA. 2005;294(13):162533. doi:10.1001/jama.294.13.1625.

6. Isseh IN, Lee R, Khedraki R, Hoffman K. A Critical Review of Hemodynamically Guided Therapy for Cardiogenic Shock: Old 
Habits Die Hard. Curr Treat Options Cardiovasc Med. 2021;23(5):29. doi:10.1007/s11936-021-00903-8.

7. Doshi R, Patel H, Shah P. Pulmonary artery catheterization use and mortality in hospitalizations with HFrEF and HFpEF: A nationally representative trend analysis from 2005 to 2014. Int J Cardiol. 2018;269:289-291. doi:10.1016/j.ijcard.2018.07.069

8. Saxena A, Garan AR, Kapur NK, O'Neill WW, Lindenfeld J, Pinney S, et al. Value of Hemodynamic Monitoring in Patients With Cardiogenic Shock Undergoing Mechanical Circulatory Support. Circulation. 2020;141(14):1184-1197. doi:10.1161/CIRCULATIONAHA.119.043080

9. Zipes DP, Libby P, Bonow RO, Mann DL, Tomaselli GF, Braunwald E. Braunwald's heart disease, a textbook of cardiovascular medicine. 11 th ed. Philadelphia: Elsevier Inc; 2019.

10. García X, Mateu L, Maynar J, Mercadal J, Ochagavía A, Ferrandiz A. Estimación del gasto cardíaco. Utilidad en la práctica clínica. Monitorización disponible invasiva y no invasiva. Medicina Intensiva. 2011; 35(9): 552 - 561. Disponible en: http://scielo.isciii.es/scielo. php?script=sci_arttext\&pid=S0210-56912011000900004\&lng=es

11. Madias C, Kimmelstiel C. (2017) Right Heart Catheterization. In: Hendel R., Kimmelstiel C. (eds) Cardiology Procedures. Springer, London. doi: 10.1007/978-1-4471-7290-1_14.

12. Mueller HS, Chatterjee K, Davis KB, Fifer MA, Franklin C, Greenberg $M A$, et al. ACC expert consensus document. Present use of bedside right heart catheterization in patients with cardiac disease. American College of Cardiology. J Am Coll Cardiol. 1998;32(3):840-864. doi:10.1016/s0735-1097(98)00327-1

13. Griffin BP, Topol EJ, editors. Manual of cardiovascular medicine. 5th ed. Philadelphia: Lippincott Williams and Wilkins; 2018.

14. Moscucci M. Grossman \& Baim's Cardiac catheterization, angiography and intervention. Philadelphia Lippincott Williams and Wilkins 9th ed. 2020.

15. Sorajja P, Lim MJ, Kern MJ. Kern's cardiac catheterization handbook. 7th ed. Oxford, Elsevier Health Sciences, 2019.

16. Kapur NK, Esposito ML, Bader Y, Morine KJ, Kiernan MS, Pham DT, et al. Mechanical Circulatory Support Devices for Acute Right Ventricular Failure. Circulation. 2017;136(3):314-326. doi:10.1161/ CIRCULATIONAHA.116.025290

17. Pellegrini $P$, Rossi A, Pasotti M, Raineri C, Cicoira M, Bonapace $S$, et al. Prognostic relevance of pulmonary arterial compliance in patients with chronic heart failure. Chest. 2014;145(5):1064-1070. doi:10.1378/chest.13-1510

18. Tampakakis E, Shah SJ, Borlaug BA, Leavy PJ, Patel HH, Miller $W L$, et al. Pulmonary Effective Arterial Elastance as a Measure of Right Ventricular Afterload and Its Prognostic Value in Pulmonary Hypertension Due to Left Heart Disease. Circ Heart Fail. 2018;11(4):e004436. doi:10.1161/CIRCHEARTFAILURE.117.004436.

19. Mazimba S, Welch TS, Mwansa H, Breathett KK, Kennedy JL, Mihalek $A D$, et al. Haemodynamically Derived Pulmonary Artery Pulsatility Index Predicts Mortality in Pulmonary Arterial Hypertension. Heart Lung Circ. 2019;28(5):752-760. doi:10.1016/j.hlc.2018.04.280

20. Kang G, Ha R, Banerjee D. Pulmonary artery pulsatility index predicts right ventricular failure after left ventricular assist device implantation. J Heart Lung Transplant. 2016;35(1):67-73. doi:10.1016/j.healun.2015.06.009.

21. D'Alto M, Dimopoulos K, Coghlan JG, Kovacs G, Rosenkranz S, Naeije R. Right Heart Catheterization for the Diagnosis of Pulmonary Hypertension: Controversies and Practical Issues. Heart Fail Clin. 2018;14(3):467-477. doi:10.1016/j.hfc.2018.03.011

22. Aschauer S, Kammerlander AA, Zotter-Tufaro C, Ristl R, Pfaffenberger $\mathrm{S}$, Bachmann A, et al. The right heart in heart failure with preserved ejection fraction: insights from cardiac magnetic resonance imaging and invasive haemodynamics. Eur J Heart Fail. 2016;18(1):71-80. doi:10.1002/ejhf.418.
23. Condon DF, Nickel NP, Anderson R, Mirza S, de Jesus Perez VA. The 6th World Symposium on Pulmonary Hypertension: what's old is new. F1000Res. 2019;8:F1000 Faculty Rev-888. Published 2019 Jun 19. doi:10.12688/f1000research.18811.1

24. Galiè N, Humbert M, Vachiery JL, Gibbs S, Lang I, Torbicki A, Simonneau G, et al. 2015 ESC/ERS Guidelines for the diagnosis and treatment of pulmonary hypertension: The Joint Task Force for the Diagnosis and Treatment of Pulmonary Hypertension of the European Society of Cardiology (ESC) and the European Respiratory Society (ERS): Endorsed by: Association for European Paediatric and Congenital Cardiology (AEPC), International Society for Heart and Lung Transplantation (ISHLT). Eur Heart J. 2016;37(1):67-119. doi:10.1093/eurheartj/ehv317

25. Ragosta, Michael. Textbook of Clinical Hemodynamics E-Book. Elsevier Health Sciences, 2017.

26. Drazner MH, Velez-Martinez M, Ayers CR, Reimold SC, Thibodeau JT, Mishkin JD, et al. Relationship of right- to left-sided ventricular filling pressures in advanced heart failure: insights from the ESCAPE trial. Circ Heart Fail. 2013;6(2):264-270. doi:10.1161/ CIRCHEARTFAILURE.112.000204

27. Vachiéry J-L, Tedford RJ, Rosenkranz S, Palazzini M, Lang I, Guazzi M, et al. Pulmonary hypertension due to left heart disease. Eur Respir J 2019; 53: 1801897

28. Simonneau G, Montani D, Celermajer DS, Denton CP, Gatzoulis MA, Krowka $M$, et al. Haemodynamic definitions and updated clinical classification of pulmonary hypertension. Eur Respir J 2019; 53: 1801913.

29. Arman T. Askari, Adrian W. Messerli. Cardiovascular Hemodynamics. USA: Human Press, 2019.

30. lyad N, Ran L, Rola K, Hoffman K. A Critical Review of Hemodynamically Guided Therapy for Cardiogenic Shock: Old Habits Die Hard. Curr Treat Options Cardio Med. 2021; 23: 29.

31. Siddiqi UA, Belkin M, Kalantari S, Kanelidis A, Miller T, Sarswat N, et al. Prognostic Role of Simultaneous Assessment of Biventricular Function Using Left Ventricular Stroke Work Index and Right Ventricular Stroke Work Index.J Heart Lung Transplant. 2021;40(4):S262.doi: 10.1016/j. healun.2021.01.747

32. Rosenkranz S, Preston IR. Right heart catheterization: best practice and pitfalls in pulmonary hypertension. Eur Respir Rev. 2015;24(138):642-652. doi:10.1183/16000617.0062-2015

33. Van Diepen S, Katz JN, Albert NM, Henry TD, Jacobs AK, Kapur NK, et al. Contemporary Management of Cardiogenic Shock: A Scientific Statement From the American Heart Association. Circulation. 2017;136(16):e232-e268. doi:10.1161/CIR.0000000000000525.

34. Tehrani BN, Truesdell AG, Sherwood MW, Desai S, Tran HA, Epps KC, et al. Standardized Team-Based Care for Cardiogenic Shock. J Am Coll Cardiol. 2019;73(13):1659-1669. doi:10.1016/j.jacc.2018.12.084.

35. Hernandez GA, Lemor A, Blumer V, et al. Trends in Utilization and Outcomes of Pulmonary Artery Catheterization in PH With and Without Cardiogenic Shock. Journal of Cardiac Failure. 2019;25(5):364-371. doi:10.1016/j.cardfail.2019.03.004.

36. Garan AR, Kanwar M, Thayer KL, Whitehead E, Zweck E, HernandezMontfort J, et al. Complete Hemodynamic Profiling With Pulmonary Artery Catheters in Cardiogenic Shock Is Associated With Lower InHospital Mortality. JACC Heart Fail. 2020;8(11):903-913. doi:10.1016/j. jchf.2020.08.012.

36. Ponikowski P, Voors AA, Anker SD, Bueno H, Cleland JGF, Coats AJS, et al. 2016 ESC Guidelines for the Diagnosis and Treatment of Acute and Chronic Heart Failure. Rev Esp Cardiol (Engl Ed). 2016;69(12):1167. doi:10.1016/j.rec.2016.11.005

37. Yancy CW, Jessup M, Bozkurt B, Butler J, Casey DE, Drazner MH, et al. 2013 ACCF/AHA guideline for the management of heart failure: a 
report of the American College of Cardiology Foundation/American Heart Association Task Force on practice guidelines. Circulation. 2013;128(16):e240-e327. doi:10.1161/CIR.0b013e31829e8776

38. Kohsaka, S, Menon V, Lowe AP, Lange M, Dzavik V, Sleeper LA, et al. (2005). Systemic Inflammatory Response Syndrome After Acute Myocardial Infarction Complicated by Cardiogenic Shock. Archives of Internal Medicine, 165(14), 1643. https://doi.org/10.1001/ archinte.165.14.1643

39. Ranka S, Mastoris I, Kapur NK, Tedford R, Rali A, Acharya P, et al. Right Heart Catheterization in Cardiogenic Shock Is Associated With Improved Outcomes: Insights From the Nationwide Readmissions Database. J Am Heart Assoc. 2021;e019843. doi:10.1161/ JAHA.120.019843.

40. Osman M, Syed M, Patel B, Munir MB, Kheiri B, Caccamo M, et al. Invasive Hemodynamic Monitoring in Cardiogenic Shock Is Associated With Lower In-Hospital Mortality. J Am Heart Assoc. 2021;10(18):e021808. doi:10.1161/JAHA.121.021808

41. Khera R, Pandey A, Kumar N, Singh R, Bano S, Golwala H, et al.Variation in Hospital Use and Outcomes Associated With Pulmonary Artery Catheterization in Heart Failure in the United States. Circ Heart Fail. 2016;9(11):e003226. doi:10.1161/CIRCHEARTFAILURE.116.003226

42. Borlaug BA. Evaluation and management of HFpEF. Nat Rev Cardiol. 2020;17(9):559-573. doi:10.1038/s41569-020-0363-2

43. McDonagh TA, Metra M, Adamo M, Gardner RS, Baumbach A, Böhm M, Burri H, et al. 2021 ESC Guidelines for the diagnosis and treatment of acute and chronic heart failure. Eur Heart J. 2021;ehab368. doi:10.1093/eurheartj/ehab368.

44. Wang W, Cheung A, Watson TJ, Ong PJ, Tcheng JE. Mechanical Complications of Acute Myocardial Infraction. In: Watson TJ, Ong PJL, Tcheng JE, eds. Primary Angioplasty: A Practical Guide. Singapore: Springer; July 14, 2018.275-287.

45. Ikuta K, Wang Y, Robinson A, Ahmad T, Krumholz HM, Desai NR. National trends in use and outcomes of pulmonary artery catheters among Medicare beneficiaries, 1999-2013. JAMA Cardiol. 2017;2(8):908-13. doi:10.1001/jamacardio.2017.1670.

46. Potapov EV, Antonides C, Crespo-Leiro MG, Combes A, Färber G, Hannan MM, et al. 2019 EACTS Expert Consensus on longterm mechanical circulatory support. Eur J Cardiothorac Surg. 2019;56(2):230-270. doi:10.1093/ejcts/ezz098. 